\title{
ESTUDO TEÓRICO DA AROMATICIDADE DOS COMPOSTOS HETERCÍCLICOS, PIRROL, FURANO E TIOFENO NO ENSINO MÉDIO
}

\author{
Juan Gustavo Chaves Paivaa; Júlia Karla Coelho Benoa; Paulo Roberto Linhares Mendes ${ }^{\text {a }}$; Allyson \\ Gabriel Melo Campos ${ }^{\mathrm{a}}$; Alexandre Costa ${ }^{\mathrm{a}^{*}}$ \\ ${ }^{a}$ Intituto Estadual de Educação, Ciência e Tecnologia do Maranhão. Centro, São Luís, MA. Brasil. \\ *E-mail para correspondência: alexandre ufma@yahoo.com.br
}

\section{Palavras-chave:}

Anéis

Heterocícliclos; Aromaticidade,; DFT.

\section{Keywords:}

Heterocyclic

Rings;

Aromaticity;

DFT.

\section{RESUMO}

A inserção do computador e das novas tecnologias no ensino de Química ou em outras ciências, pode ser um importante recurso para facilitar o processo de construção de conhecimento. Tendo isso em vista, o objetivo deste estudo foi compreender a aromaticidade dos compostos heterocíclicos pirrol, furano e tiofeno através de cálculos teóricos usando programas computacionais como ferramentas metodológicas para o ensino deQuímica, a partir da perspectiva dos professores e alunos. Todo o trabalho foi desenvolvido por um grupo de Iniciação à Química Teórica formado por quatro alunos do $3^{\circ}$ ano do Curso Técnico em Informática do IEMA UP São Luís-MA. Os critérios de aromaticidade energéticos, estruturais ou geométricos e magnéticos foram usados no respectivo trabalho. A pesar das dificuldades iniciais no uso dos programas computacionais de química quântica, os alunos desenvolveram de forma satisfatória os comandos necessários para realização dos cálculos teóricos para o estudo da aromaticidade. O uso dos programas de química teórica no ensino de química, podem servir como mecanismo de apoio e recurso didático auxiliar à assimilação dos conteúdos, facilitando assim, o entendimento dos alunos nos assuntos que são considerados abstratos e de difícil compreensão.

\section{ABSTRACT}

The inserting of computers and new technologies in teaching Chemistry or other sciences can be an important resource to facilitate the process of knowledge construction. With this in mind, the aim of this study was to understand the aromaticity of the heterocyclic compound s pyrrole, furan, and thiophene through theoretical calculations using computer programs as methodological tools for teaching Chemistry, from the perspective of teachers and students. All the work was developed by a group of Initiation to Theoretical Chemistry formed by four students of the 3rd year of the Technical Course in Informatics at IEMA UP São Luís-MA. The energetic, structural or geometric and magnetic aromaticity criteria were used in the respective work. In spite of the initial difficulties in the use of quantum chemistry computer programs, students have developed satisfactorily the commands necessary to carry out theoretical calculations for the study of aromaticity. The use of theoretical chemistry programs in the teaching of chemistry can serve as a support mechanism and didactic resource to help assimilate the contents, thus facilitating students' understanding of subjects that are considered abstract and difficult to understand.

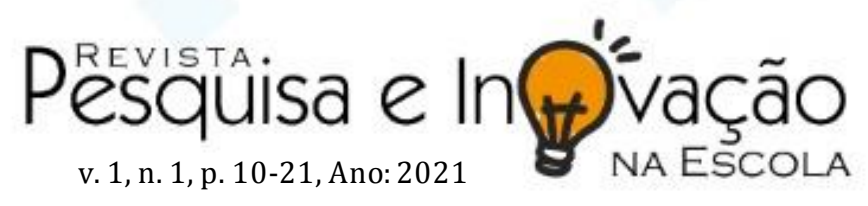




\section{INTRODUÇÃO}

A Química, embora seja considerada atrativa, é muitas vezes também considerada de difícil compreensão. Os softwares de computad or podem tornar o ensino de Química mais fácil e manter os alunos mais participativos (YU; CHEN, 2012). Atualmente os softwares de química quântica são cada vez mais usados no ensino (ALLOUC, 2011). Na Química Quântica um dos programas mais usados no meio científico é o Gaussian (TRSIC; PINTO, 2009, p. 108) que utiliza as leis da mecânica quântica para prevê as energias, propriedades estruturas, propriedades vibracionais e outras propriedades dos sistemas moleculares. Este foi o software usado para o estudo da aromaticidade de três anéis heterocíclicos de cinco membros.

O objetivo deste trabalho foi estudar a aromaticidade dos anéis heterocíclicos contendo um heteroátomo: nitrogênio, oxigênio, enxofre, chamados pirrol, furano e tiofeno, respectivamente. Esses compostos são similares ao ânion aromático ciclopentadienil, em que o CH é trocado por NH, O e S (MONDINO, 2014, p. 62).

Por que estudar os heteroátomos aromáticos? Porque a aromaticidade é a pedra angular da química dos heterocíclicos, sejam eles de cinco ou seis membros, monocíclicos ou fundidos ou com vários heteroátomos. A presença de um ou mais heteroátomos em um anel aromático modifica os critérios de reatividade, tautomeria, propriedades magnéticas e energia de ressonância, diferenciando-os dos aromáticos carbocíclicos na forma como reagem e como se relacionam com outras substâncias (MONDINO, 2014, p. 61).

A aromaticidade é um conceito fundamental na Química Orgânica, que descreve a estabilidade especial de moléculas ciclicamente conjugadas com um certo número de elétrons (SOLÀ, 2018). Por outro lado, a antiaromaticidade descreve uma desestabilização. A regra de Hückel (HÜCKEL, 1931, 1932, 1937) pode ser usada para decidir se uma molécula é aromática ou antiaromática. De acordo com essa regra, uma molécula é aromática somente se possuir um sistema de conjugação monocíclico e plano que contenha um total de $(4 n+2)$ elétrons $\pi$, em que $\mathrm{n}$ é número inteiro. As moléculas com (4n) elétrons $\pi$, além de não serem aromáticos, são antiaromáticas. Se o composto cíclico não possui $(4 n+2)$ elétrons $\pi$ e nem ( $4 n)$ elétrons $\pi$, esses são denominados não-aromáticos. É importante frisar que a aromaticidade não é uma propriedade exclusiva de sistemas ditos usuais, ou seja, sistemas cíclicos conjugados que exibem deslocalização de elétrons, mas também é considerada para sistemas ditos não convencionais. Desde a regra inicial deHückel, várias regras de aromaticidade foram propostas (FEIXAS et al., 2016). O fenômeno da aromaticidade é de fato complexo e multidimensional, ou seja, apesar de não ser uma observável física, pode ser caracterizado através de diferentes

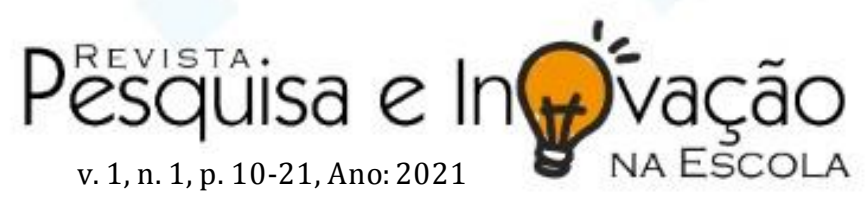


critérios. Os critérios tradicionais de aromaticidade incluem comportamento químico (menor reatividade), características estruturais (planaridade e tendências de comprimento de ligação iguais), energia (estabilidade) e propriedades espectroscópicas (UV, mudanças químicas de prótons, exaltação da suscetibilidade magnética) (MERINO; HEINE; SEIFERT, 2004).

\section{METODOLOGIA}

Para atingir os objetivos propostos no trabalho, foram estudadas a aromaticidade de três compostos de anéis de cinco membros, pirrol, furano e tiofeno. Essas estruturas foram comparadas à aromaticidade do anel de seis membros do benzeno. Os cálculos foram efetuados pelo código de química quântica Gaussian09 (FRISCH et al., 2009). Todas as moléculas investigadas foram otimizadas empregando-se o método Teoria do Funcional da Densidade (sigla em inglês DFT), utilizando o funcional híbrido B3LYP (POPLE et al., 1987; BECKE, 1993; LEE, 1988). O conjunto de base 6-311(d,p) foi usado para os átomos (C, N, S e H). Os cálculos das otimizações das geometrias e frequências vibracionais foram realizados com a mesma função de base 6-311G(d,p). As frequências vibracionais das estruturas otimizadas foram usad as para obtenção da energia vibracional do ponto zero (sigla em inglês ZPE) (Curtiss, 1991). A avaliação das transferências de carga sobre os átomos foi obtida pela análise da população de Mulliken (MULLIKEN, 1955). Os resultados foram visualizados com o programa Gauss View 5.0 (DENNINGTON, 2009).

Os critérios mais aceitos de aromaticidade podem ser divididos em energéticos, estruturais ou geométricos e magnéticos. No presente trabalho, foram adotados os três critérios para considerar a aromaticidade dos compostos. Suas geometrias otimizadas em nível B3LYP/6-311G(d,p) são mostrad as na Figura 1.

Figura 1: Estruturas otimizadas do benzeno, pirrol, furano e tiofeno em nível B3LYP/6$311 G(d, p)$.
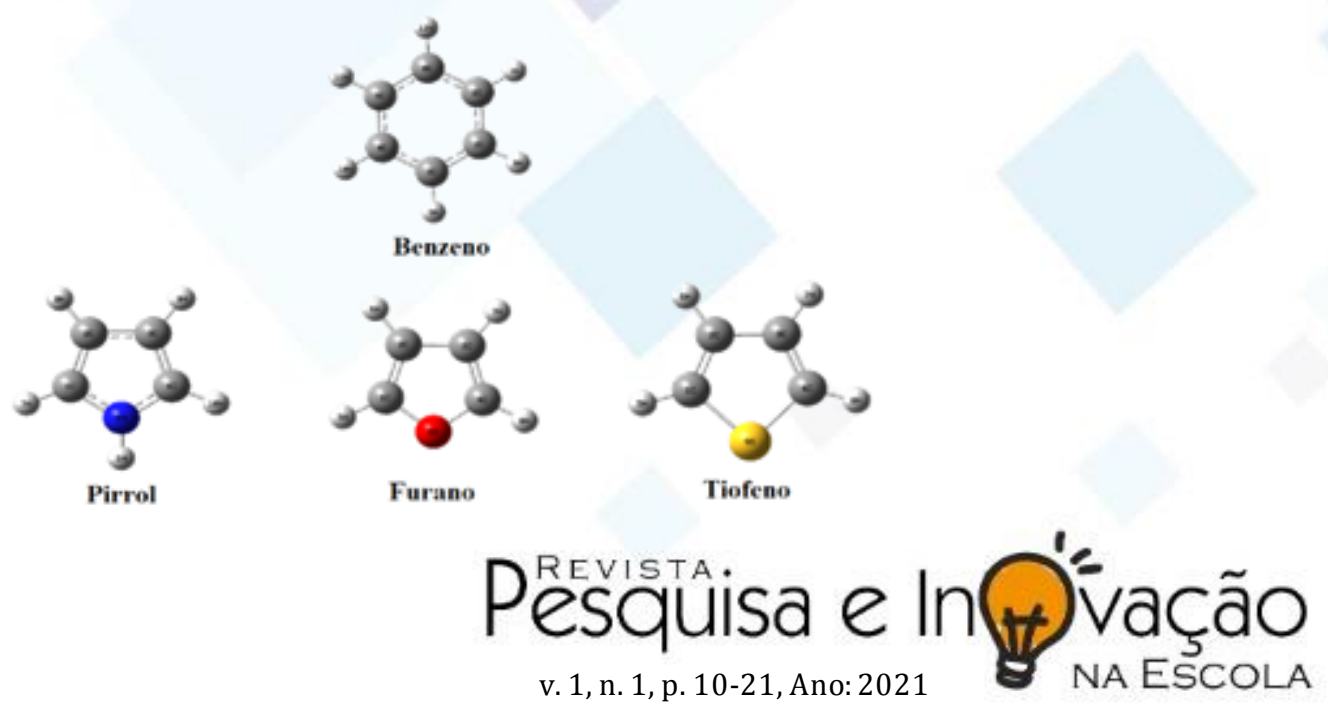
Os critérios energéticos estão baseados nos calores de reação, energias de ressonância e cálculos teóricos. Os critérios estruturais usam os dados do comprimento de ligação e ordem de ligação, dentre eles, os dois índices amplamente usados são: o modelo de oscilad or harmônico para a aromaticidade (HOMA) (KRUSZEWSKI; KRYGOWSKI, 1972; KRYGOWSKI, 2014) e o índice de Bird (BIRD, 1985); para esses cálculos usamos o software Multiwfn Versão 3.7 (LU; CHEN, 2012). O HOMA é um dos índices de aromaticidade mais simples, mais bemsucedidos e mais amplamente usados (KRUSZEWSKI; KRYGOWSKI, 1972; KRYGOWSKI, 2014). Ele abrange o aspecto geométrico da aromaticidade em uma fórmula na qual o comprimento da ligação do benzeno é o padrão interno de aromaticidade perfeita. Quanto mais diferente do benzeno e quanto mais desiguais e alternadas forem as ligações, menor será o índice HOMA do anel analisado, denotando o quão menos geometricamente aromático ele é. $\mathrm{O}$ índice HOMA pode ser expresso da seguinte fórmula:

$$
\text { HOMA }=1-n i=1 n(R R e f-R i, j) 2
$$

Onde:

$\boldsymbol{n}$ é o número de ligações consideradas para no anel;

$j$ denota o átomo próximo ao átomo $i$; e

$\boldsymbol{\alpha}$ é um fator de normalização para as ligações $\mathrm{C}-\mathrm{C}$ e $\mathrm{C}-\mathrm{N}, \mathrm{C}-\mathrm{O}$ e $\mathrm{C}-\mathrm{S}\left(\alpha=257,7 \AA^{-2}, \alpha=93,5\right.$ $\AA^{-2}, \alpha=157,4 \AA^{-2}$ e $\alpha=94,1 \AA^{-2}$ ), respectivamente escolhidas de tal forma que HOMA=0 para um modelo de sistema não aromático, e HOMA=1 para um sistema com todas as ligações iguais a um valor ideal $R_{R e f}(1,388,1,334,1,265,1,677 \AA$ para ligações $\mathrm{C}-\mathrm{C}$ e C-N, C-O e C-S, respectivamente) assumido como alcançado para sistemas totalmente aromáticos;

$\boldsymbol{R}_{i}$ representam o $i$ ésimo comprimento da ligação no anel analisado.

O Índice de Bird (BIRD, 1985) é outra quantidade baseada em geometria destinada a medir aromaticidade e pode ser calculada pela fórmula:

$$
\mathrm{I}=100[1-(\mathrm{VVK})]
$$

Onde:

$$
V=100 N i(N i, j-N) 2 n \quad N i, j=a R i, j-b
$$

Na fórmula, $i$ dá um ciclo em todas as ligações do anel, $j$ denota o átomo próximo ao átomo $i$. $n$ é o número total de ligações considerados. $N$ denota a ordem da ligação Gordy, $\mathrm{N}$ é o valor médio dos valores $N . R_{i, j}$ é o comprimento da ligação. $a$ e $b$ são parâmetros predefinidos,

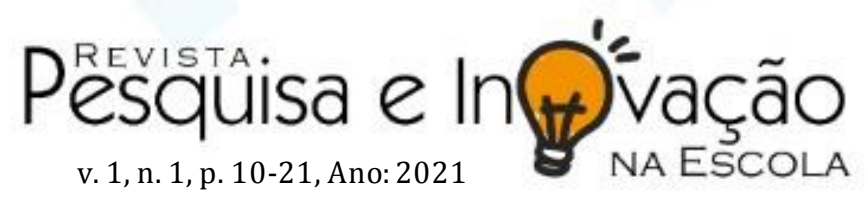


respectivamente, para cada tipo de ligação. $V_{K}$ é a referência $V$ pré-determinada, para anéis de cinco e seis membros o valor é 35 e 33,2, respectivamente. Quanto mais o índice de Bird estiver próximo de 100, mais forte é a aromaticidade. $a$ e $b$ disponíveis incluem C-C, C-N, C-O, C-S; para outro tipo de ligação.

O deslocamento químico independente de núcleo (sigla em inglês NICS), (SCHLEYER et al., 1996) é um índice de aromaticidade com base magnética, que está relacionado às propriedades magnéticas das moléculas conforme mostra a Figura 2. Em 1996, propuseram esse novo índice magnético de aromaticidade (SCHLEYER et al., 1996). O NICS é descrito como o valor negativo dablindagem isotrópica total calculada em um ponto escolhido no espaço (SCHLEYER et al., 1996). Um valor de NICS negativo significativo (blindado magneticamente) dentro de um anel ou gaiola especifica indica a ocorrência de uma corrente de anel diatrópico induzida ou "aromaticidade", enquanto um valor de NICS positivo (sem proteção magnética) indica uma corrente paratrópica induzida ou antiaromaticidade (PATCHKOVSKII; THIEL, 2000).

Atualmente, o índice de aromaticidade do NICS é amplamente utilizado, e muitos estudos $a b$ initio e funcionais de densidade provaram que o NICS é um bom critério de aromaticidade de hidrocarbonetos aromáticos policíclicos (sigla em inglês PAHs) (PATCHKOVSKII; THIEL, 2000). Em geral, concorda bem com outros critérios de aromaticidade (PORANNE; STANGER, 2015).

Figura 2: NICS(0) calculado com o átomo fictício colocado no mesmo plano que o anel, e o NICS(1) calculados com uma distância de 1 Å perpendicular ao anel.

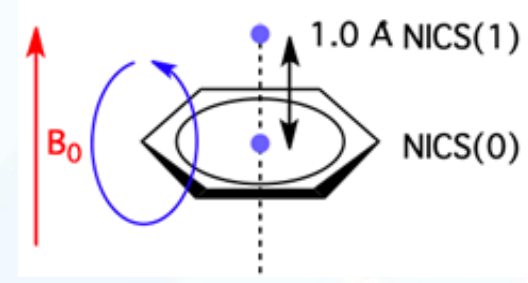

\section{RESULTADOS E DISCUSSÃO}

O estudo iniciou-se com a pesquisa da conformação mais estável para as moléculas de benzeno, pirrol, furano e tiofeno, através dos cálculos de otimização estrutural. Assim, os alunos puderam entender a funcionalidade do programa ao realizar os cálculos para se atingir a energia mínima para os compostos. Dessa forma, entende-se que o conhecimento se consolida, ao passo

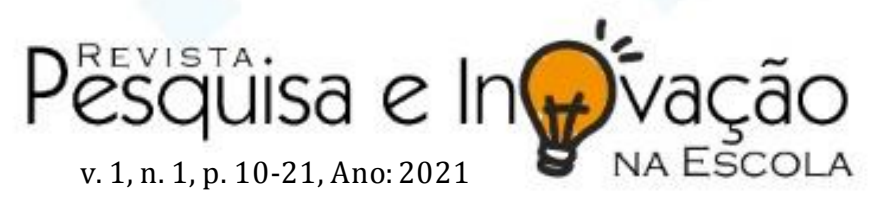


que o aluno percebe as mudanças nos comprimentos e ângulos de ligação para se chegar ao estado mais estável das moléculas.

A Tabela 1 apresenta para as moléculas suas simetrias, os valores das energias totais e seus momentos de dipolos elétricos experimentais e calculados usando o nível de cálculo B3LYP/6-311G(d,p). Nota-se que todos os compostos apresentaram geometria planar, o que indica uma característica de um composto aromático; consequentemente, todos apresentaram a mesma simetria $\mathrm{C}_{1}$. Observamos ainda, que a energia total do tiofeno se diferencia consideravelmente dos demais compostos. Nesse sentido, os cálculos do tipo B3LYP/6$311 \mathrm{G}(\mathrm{d}, \mathrm{p})$ apresentaram os menores valores de energia seguindo a respectiva ordem de estabilidade energética: tiofeno>benzeno $>$ furano $>$ pirrol.

O momento de dipolo elétrico $\boldsymbol{\mu}$ de uma distribuição de carga é definido por $=$ iQiri onde ri é o vetor da origem (escolhida de forma arbitrária) até a carga Qi. A demonstração dessa afirmativa é apresentada em (PECK, 1953, p.29). Assim como o pirrol, o furano e tiofenos apresentam híbridos de ressonância com cinco estruturas. Em comparação com o furano e o tiofeno, o momento de dipolo do pirrol inesperadamente tem a direção oposta conforme mostra a Figura 3a. Isso é surpreendente, já que o polo positivo do dipolo do pirrol está localizado no nitrogênio, embora o nitrogênio seja mais eletronegativo do que o carbono.

As cargas parciais negativas nos átomos de carbono do pirrol geram cargas parciais positivas no átomo de nitrogênio, desencadeando uma polarização, que é compensada pelo ef eito indutivo em sentido contrário, assim, o efeito da ressonância na distribuição de elétrons obviamente supera o efeito que a maior eletronegatividade do nitrogênio tem na distribuição de elétrons obtendo-se um momento de dipolo de grande magnitude de $\mu=1,94 \mathrm{D}$. No furano e no tiofeno, o momento de dipolo é influenciado pela ressonância da mesma forma que no pirrol. No entanto, o efeito da ressonância obviamente não é suficiente para exceder o efeito da eletronegatividade do heteroátomo $\mathrm{O}$ e $\mathrm{S}$. Assim, o polo negativo está localizado nos heteroátomos $\mathrm{O}$ e $\mathrm{S}$, devido à maior ressonância, os momentos de dipolos do furano e do tiofeno são menores $\mu=0,62$ e $0,52 \mathrm{D}$.

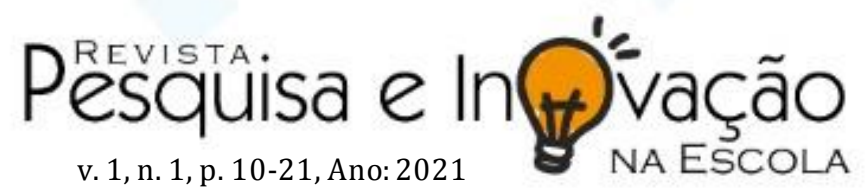


Tabela 1: Compostos: simetria, energia total $\left(E_{\mathrm{T}}\right.$, em hartrees) e momento de dipolo ( $\mu$, em debyes).

\begin{tabular}{|c|c|c|c|c|c|}
\hline Compostos & Simetria & $\mathbf{E}_{\mathrm{T}}$ & $\boldsymbol{\mu}^{\mathrm{a}}$ & $\boldsymbol{\mu}^{\mathrm{b}}$ \\
\hline Benzeno & $\mathrm{C}_{1}$ & $-232,30854445$ & - & 0,00 \\
Pirrol & $\mathrm{C}_{1}$ & $-210,22608145$ & 1,81 & 1,94 \\
Furano & $\mathrm{C}_{1}$ & $-230,08341163$ & 0,70 & 0,62 \\
Tiofeno & $\mathrm{C}_{1}$ & $-553,06968444$ & 0,51 & 0,56 \\
\hline
\end{tabular}

Valores experimentais dos momentos de dipolo do benzeno, pirrol, furano e tiofeno (Mondino, 2014, p. 66,74,81). ${ }^{\circ}$ Valores teóricos dos momentos de dipolo calculados no trabalho.

A capacidade de ligação de uma molécula depende da carga eletrônica nos átomos. A eletronegatividade, $\chi$ (chi), de um elemento é a capacidade que um átomo de um elemento tem de atrair os elétrons para ele quando faz parte de um composto (Atkins et al., 2008, p.52). Analisando-se a Figura 3a, constata-se que as cargas atômicas de Mulliken reproduzem bem a eletronegatividade para o átomo de nitrogênio $\mathrm{Q}_{\mathrm{N}}<0$, oxigênio $\mathrm{Q}_{\mathrm{O}}<0$ e enxofre $\mathrm{Q}_{\mathrm{s}}>0$. $\mathrm{A}$ acentuada diferença de carga entre os átomos NC, OC e SC nos compostos pirrol, furano e tiofeno, implicam que as ligações são de natureza covalente polar. Os resultados, podem ser melhor representados na forma gráfica, pois será possível dizer a mudança na distribuição de carga apenas pela mudança na orientação da molécula como mostra a Figura 3b.

Figura 3: Cargas atômicas nas moléculas de: (a) benzeno, pirrol, furano e tiofeno e a direção do momento de dipolo; (b) mostrada através de escala de cores.
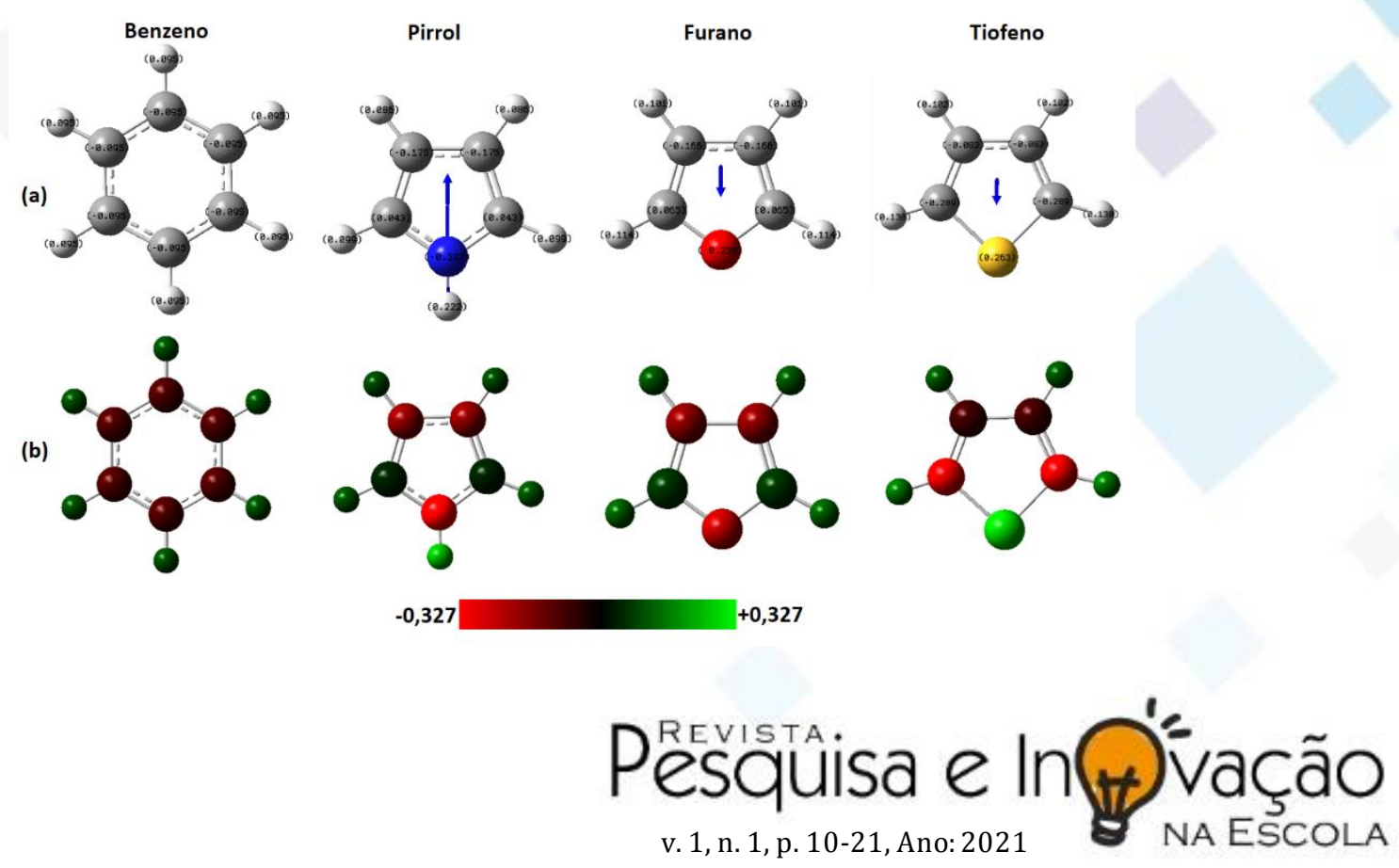
A Figura 4 apresenta a densidade de elétrons no qual a superfície de potencial eletrostático foi mapeada para os compostos benzeno, pirrol, furano e tiofeno a partir dos cálculos de otimização. Por meio dos mapas de potencial os alunos puderam entender que estas superfícies representam o tamanho, forma, densidade de carga, e o local de reatividade química das moléculas. O vermelho representa as regiões de potencial eletrostático fortemente negativo, o azul representa regiões de potencial eletrostático fortemente positivo. Observa-se que a densidade eletrônica está simetricamente distribuída no benzeno, o que é confirmado pela análise das cargas de Mulliken (Figura $3^{\mathrm{a}}$ ) e o seu momento de dipolo nulo como mostra a Tabela 1. No entanto, para os compostos pirrol, furano e tiofeno nos anéis de cinco membros, mostram como o átomo de $\mathrm{N}$ é pobre em elétron (menos vermelho), enquanto os átomos $\mathrm{O}$ e $\mathrm{S}$ do são mais ricos em elétrons (mais vermelho).

Figura 4: Mapa do potencial eletrostático do benzeno, pirrol, furano e borazina tiofeno.

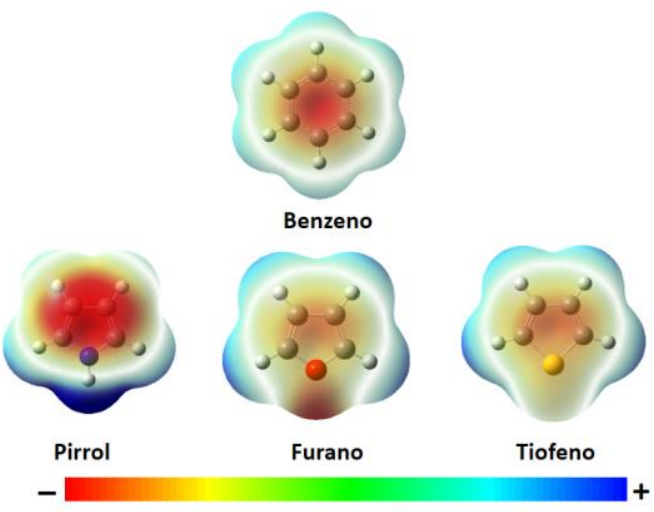

A fim de estudar a aromaticidade do benzeno, pirrol, furano e tiofeno, os alunos usaram o índice de energia de ressonância estimad o por vários métodos (Mondino, 2014, p. 62) como podemos observar na Tabela 2. Eles também realizaram os cálculos teóricos do índice estrutural HOMA, índice de Bird de aromaticidade e o NICS como mostra a Tabela 3. Segundo CORMINBOEUF et al. (2004) os valores isotrópicos de $\left(\right.$ NICS $\left._{\text {iso }}\right)$ no centro do anel contêm amplas influências do sistema $\sigma$ e os três componentes principais do tensor de NICS. Por outro lado, o NICS $\mathrm{zz}_{\mathrm{zz}}$ fornece principalmente as contribuições $\pi$ a grandes distâncias do centro do anel. Assim, os NICS foram calculados acima dos centros dos anéis de cinco e seis membros, variando as distâncias de 0,0 a 1,0 ̊. Observando os dados, percebemos que nos três critérios de aromaticidade adotados no trabalho, a ordem de aromaticidade é: benzeno $>$ tiofeno $>$ pirrol $>$ furano.

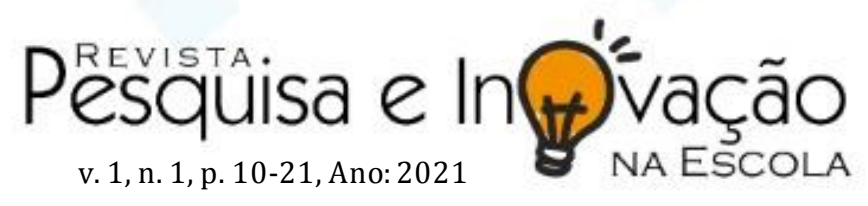


Tabela 2: Índice de energia de ressonância estimados por vários métodos (Mondino, 2014, p.62).

\begin{tabular}{|c|c|c|c|}
\hline Compostos & ${ }^{\mathrm{a}}$ ERE(Kjmol-1) & ${ }^{\prime D R E}\left(\right.$ Kjmol'-1) $^{-1}$ & 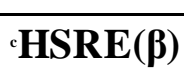 \\
\hline Benzeno & 25,0 & 15,77 & 0,065 \\
\hline Pirrol & 15,1 & 5,86 & 0,039 \\
\hline Furano & 11,3 & 3,01 & 0,007 \\
\hline Tiofeno & 20,3 & 4,52 & 0.032 \\
\hline
\end{tabular}

'ERE: energia empírica de ressonância; ${ }^{D} D R E$ : energia de ressonância de Dewar; ${ }^{H}$ HSE: energia de ressonância de Hess-Shaad.

Ressaltamos ainda, que tanto no HOMA quanto no NICS o pirrol apresenta maior aromaticidade que o tiofeno. Os valores negativos de $\operatorname{NICS}(0)_{\text {iso, }}, \operatorname{NICS}(0)_{z}, \operatorname{NICS}(1)_{\text {iso }}$ e NICS $(1)_{z z}$ confirmaram a aromaticidade e a existência de correntes de anel diamagnético diatrópico em todos os compostos, como mostra a Tabela 4. Nota-se que apenas os valores de NICS(1) $)_{\text {iso }}$ não descreve a mesma tendência dos cálculos NICS $_{i s o}$ e NICS ${ }_{z z}$ dos sistemas estudados.

Tabela 3: Índice estrutural HOMA e índice Bird de aromaticidade e valores do NICS (em ppm) a 0 , e 1 A acima dos anéis.

\begin{tabular}{|c|c|c|c|c|c|c|}
\hline Compostos & HOMA & $I_{\mathrm{B}}$ & $\operatorname{NICS}(0)_{\text {во }}$ & $\operatorname{NICS}(1)_{\mathrm{ko}}$ & $\operatorname{NICS}(0)_{n}$ & $\operatorname{NICS(1)_{u}}$ \\
\hline Benzeno & 1,000 & 100 & $-8,89$ & $-11,13$ & $-14,49$ & $-29,33$ \\
\hline Pirrol & 0,860 & 65 & $-14,81$ & $-10,99$ & $-12,11$ & $-30,94$ \\
\hline Furano & 0,194 & 42 & $-13,05$ & $-10,13$ & $-8,50$ & $-27,39$ \\
\hline Tiofeno & 0,745 & 56 & $-13,87$ & $-11,27$ & $-9,24$ & $-28,08$ \\
\hline
\end{tabular}

\section{CONCLUSÃO}

A análise das cargas de Mulliken dos compostos pirrol, furano e tiofeno reproduzem bem a eletronegatividade para o átomo de nitrogênio $\mathrm{Q}_{N}<0$, oxigênio $\mathrm{Q}_{0}<0$ e enxofre $\mathrm{Q}_{\mathrm{s}}>0$. Por meio dos mapas de potencial eletrostáticos das moléculas os alunos puderam analisar como a distribuição eletrônica variou nas moléculas.

O uso dos três critérios de aromaticidade: energético (índice de energia de ressonância), estrutural (HOMA e índice Bird de aromaticidade) e magnético (NICS), foram e promissores 
para a determinação da aromaticidade dos compostos. Além dos conceitos de aromaticidade e antiaromatidade, já estabelecidos nos livros textos usados nos encontros, destaca-se o crescente interesse dos alunos envolvidos no grupo, na busca de um maior aprofundamento no estudo de outros compostos aromáticos.

\section{AGRADECIMENTOS}

Este estudo teve apoio da Gestão Geral (Jhonatan Matos Camilo) e Gestão Pedagógica (Cleriston Araujo Silva) do Instituto Estadual de Educação, Ciência e Tecnologia do Maranhã (IEMA) da Unidade Plena São Luís Centro. Todo o trabalho não poderia ter se realizado sem a criação grupo de Iniciação à Química Teórica no IEMA UP São Luís Centro.

\section{REFERÊNCIAS}

ALLOUCHE, A.-R. Gabedit - A graphical user interface for computational chemistry softwares. Journal of computational chemistry, United States, v. 32, n.1, p. 174-182, May. 2011.

ATKINS, P. W.; OVERTON, T.; ROURKE, J.; WELLER, M.; ARMSTRONG, F. Química Inorgânica, 4a Edição, Artmed Editora S.A, 2008.52 p.

BECKE, A. D. Density-functional thermochemistry.III. Journal of Physical Chemistry, United States, v. 98, n. 7, p. 5648-5652, Dec. 1993.

BIRD, C. W. A new aromaticity index and its application to five-membered ring heterocycles. Tetrahedron, United Kingdom, v. 4, n. 7, p. 1409-1414, Feb. 1985.

CURTISS, L. A.; RAGHAVACHARI, K.; TRUCKS, G. W.; POPLE, J. A. Gaussian-2 theory for molecular energies of first- and second-row compounds. The Journal of Chemical Physics, United States, v. 94, n. 11. p. 7221-7230, Feb. 1991.

CHEN, Z.; WANNERE, C. S.; CORMINBOEUF, C.; PUCHTA, R.; SCHLEYER, P. v. R. Nucleus-independent chemcal shifts (NICS) as an aromaticity criterion. Chemical Reviews, United States, v. 105, n. 10, p. 3842-3888, Sept. 2005.

CORMINBOEUF, C.; HEINE, T.; SEIFERT, G.; SCHLEYER, P. v. R.; WEBER, J. Ind uced magnetic fields in aromatic [n]-annulenes-interpretation of NICS tensor components. Physical Chemistry Chemical Physics, United Kingdom, v. 6, n. 2, p. 273-276, Dec. 2004.

DENNINGTON, R.; KEITH, T.; MILlAM, J. Gauss View Version 5, Semichem Inc., Shawnee Mission, 2009.

KRUSZEWSKI, J.; KRYGOWSKI, T. M. Definition of aromaticity basing on the harmonic oscillator model. Tetrahedron Letters, United Kingdom, v. 13, n. 36, p. 3839- 3842, 1972.

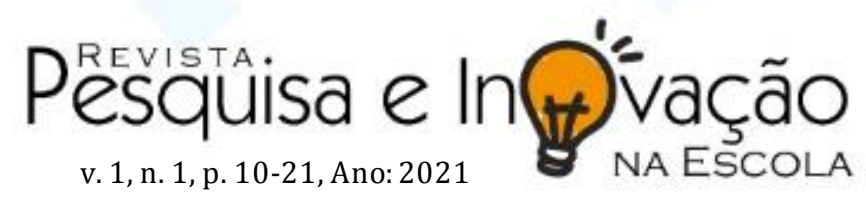


KRYGOWSKI, T. M.; SZATYLOWICZ, H.; STASYUK, O. A.; DOMINIKOWSKA, J.; PALUSIAK, M. Aromaticity from the Viewpoint of Molecular Geometry: Application to Planar Systems. Chemical Reviews, United States, v. 114, n. 12, p. 6383-6422, Apr. 2014.

LEE, C.; YANG, W.; PARR, R.G. Development of the Colle-Salvetti correlation-energy formula into a functional of the electron density. Physical Review B, United States, v. 37, n. 2, p. 785-789, Jan. 1988.

LU, T.; CHEN, T. Multiwfn: a multifunctional wavefunction analyzer. Journal of Computational Chemistry, United States, v. 33, n. 5, p. 580-592, Dec. 2012.

FEIXAS F.; MATITO, E.; POATER J.; SOLÀ M. Rules of Aromaticity. CHAUVIN, R.; LEPETIT, C.; SILVI, B.; ALIKHANI, E. In Applications of Topological Methods in Molecular Chemistry, Berlin, Eds Springer, 2016. 321-336 p.

FRISCH, M. J. et al. Gaussian 09, Revision D.01. Gaussian, Inc.: Wallingford CT, 2009.

HÜCKEL, E. Quanstentheoretische Beiträge zum Benzolproblem I. Quantentheorie der induzierten Polaritäten. Zeitschrift für Physik, Germany, v. 70, n. 3 p. 204-286, Apr. 1931.

HÜCKEL, E. Quanstentheoretische Beiträge zum Benzolproblem II. Quantentheorie der induzierten Polaritäten. Zeitschrift für Physik, Germany, v. 72, n. 5, p. 310-337, Aug. 1931.

HÜCKEL, E. Quantentheoretische Beiträge zum Problem der aromatischen und ungesättigten Verbindungen. III. Zeitschrift für Physik, Germany, v. 76, n. 9, p. 628-648, Apr. 1932.

HÜCKEL, E. The theory of unsaturated and aromatic compounds. Z. Elektrochemie, Germany, v.43, p. 752-788, 827-849, 1937.

MONDINO, M. G. Compostos Heterocíclicos: Estudos e Aplicações Sintéticas, São Paulo: Editora Atheneu, 2014.61, 62, 66, 74, 81 p.

MERINO, G.; HEINE, T.; SEIFERT, G. The induced magnetic field in cyclic molecules. Chemistry European Journal, France, v.10, n. 17, p. 4367-4371, Aug. 2004.

MULLIKEN, R. S. Electronic population analysis on LCAO-MO molecular wave functions. The Journal of Chemical Physics, United States, v. 23, n. 10, p. 1833-1840, Dec. 1955.

POPLE, J.A.; HEAD-GORDON, M.; RAGHAVACHARI, K. Quadratic configuration interaction. A general technique for determining electron correlation energies. Journal of Chemical Physics, United States, v. 87, n. 10, p. 5968-5976, Aug. 1987.

PATCHKOVSKII, S.; THIEL, W. Nucleus-independent chemical shifts from semiempirical calculations. Journal of Molecular Modeling, Germany, v. 6, n. 2, p. 67-75, Feb. 2000.

PORANNE, R. G.; STANGER, A. Magnetic Criteria of Aromaticity. Chemical Society Reviews, United Kingdom, v. 44, n. 18, p. 6597-6615, June. 2015.

PECK, E. R. Electricity and Magnetism, McGraw-Hill, 1953. 29 p.

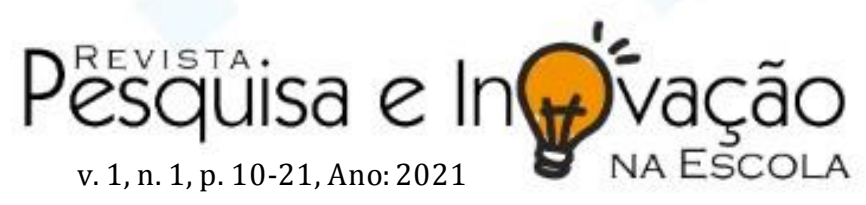


SOLÀ, M. Connecting and Combining Rules of Aromaticity. Towards a unified theory of aromaticity. WIREs Computational Molecular Science, United States, v. 9, n. 4, p. 1404, Dec. 2018.

SCHLEYER, P. v. R.; MAERKER, C.; DRANSFELD, A.; JIAO, H.;HOMMES, N. J. R. v. E. Nucleus-Independent Chemical Shifts: A Simple and Efficient Aromaticity Probe. Journal of the American Chemical Society, United States, v. 118, n. 26, p. 6317-6318, July. 1996.

TRSIC. M.; PINTO, M. F. S. Química quântica: fundamentos e aplicações. Barueri, SP: Manole, 2009. 108 p.

YU, W.; CHEN, L. The Application of Computer Softwares in Chemistry Teaching. International Journal of Education and Management Engineering, Hong Kong SAR, v. 2, n. 12, p. 73-77, Mar. 2012. 